\title{
Gold and the Beginnings of Physical Metallurgy
}

\author{
THE PIONEER WORK OF ROBERTS-AUSTEN
}

\author{
J. C. Chaston
}

In 1888, William Chandler Roberts-Austen, who was then Chemist and Assayer of the Royal Mint in London, published in the Philosophical Transactions of the Royal Society a paper on "Certain Mechanical Properties of Metals Considered in Relation to the Periodic Law".

This was, in essence, simply a study of the effects of small additions of impurities on the strength and malleability of gold; but the results seemed to many people to open up so promising an approach to a fundamental theory of metals that the Institution of Mechanical Engineers, on the recommendation of Dr Anderson, the Director-General of Ordnance Factories, was prompted to set up the famous Alloys Research Committee, a move that really initiated the science of physical metallurgy and that eventually led to the establishment in 1901 of the Department of Metallurgy at the National Physical Laboratory.

At the time, several instances of the quite remarkable effects of small alloying additions to metals were well known-in particular the effects of carbon additions to iron-but the means by which the effects were produced remained a complete mystery.

It had occurred to Roberts-Austen that the mode of action of these small quantities might be "under the control of the law of periodicity" which was then receiving a good deal of attention in scientific circles. This "law" stated that "the properties of the compounds of the elements are a periodic function of the atomic weights of their constituent elements". It

\section{Sir William Chandler Roberts-Austen 1843-1.902}

Born William Chandler Roberts, he added the name of an uncle Austen in 1885. After studying at the Royal School of Mines he was appointed Chemist of the Mint in 1870, and coupled this with his later appointment, in 1880, as the successor to John Percy as Professor of Metallurgy at the Royal School of Mines. Knighted in 1899, he continued as Acting Deputy Master of the Mint-where most of his research was carried out-until his death. The quality of his pioneering work in physieal metallurgy, beginning with a study of gold alloys, ensured its immediate recognition and its embodiment into all subsequent works on the subject should be borne in mind that there was a good deal of uncertainty concerning the difference between chemical compounds and alloys. Further, the nature and extent of allotropy in certain metals was a lively topic of speculation and enquiry.

To test this idea, he selected gold as the most suitable basis for experiment. "Gold" he wrote "can be more readily brought to a high degree of purity than any other metal; the accuracy of the results of the experiments are not likely to be disturbed by the oxidation of the gold or by the presence of occluded gas: it possesses considerable ductility and tenacity: and the amount of the metallic or other impurity added to the precious metal can be determined with rigorous accuracy".

Roberts-Austen accordingly made arrangements for a series of 17 gold alloys to be made in the Assay Department of the Royal Mint. The work was under the care of "Mr Groves whose careful manipulation and great experience in melting the precious metals were", Roberts-Austen wrote, "of much service to me. The pure gold was in all cases melted under a pure form of charcoal; a certain amount of the metallic impurity to be added was carefully weighed on a

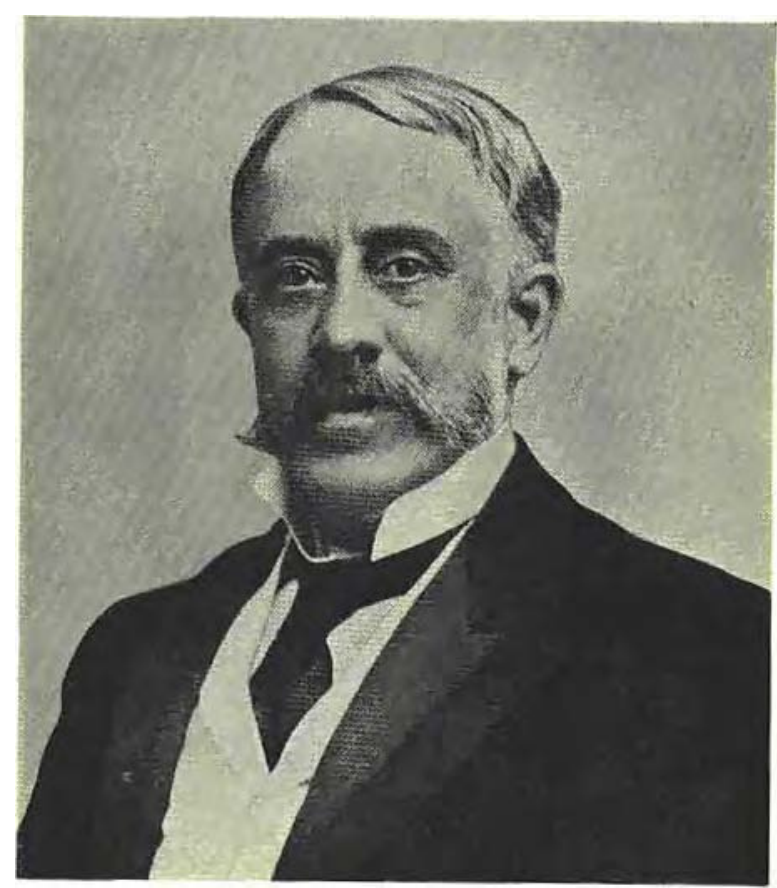



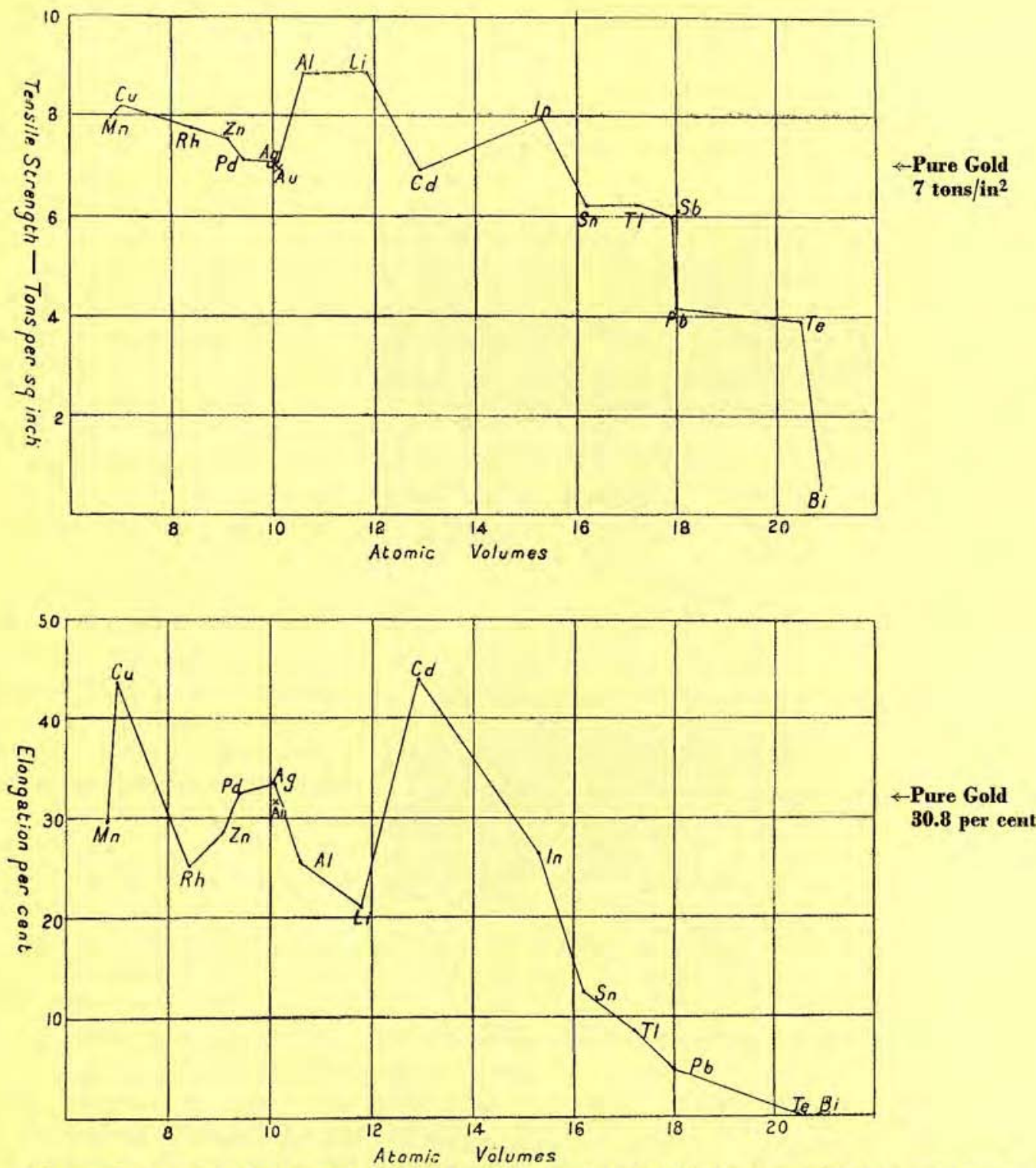

The graphs from Roberts-Austen's paper of 1838, showing the effects of small alloying additions on the tensile strength and elongation of gold plotted against the calculated atomic volume of the added elements. The values for pure gold can be seen at 7 tons $/$ in $^{2}$ and $30.8 \%$. Arising from this work, Roberts-Austen was appointed by the Alloys Research Committee of the Institution of Mechanical Engineers to determine whether the principle that the larger the volume of the added atoms the greater the effect would apply also to the properties of iron, copper and lead alloys

delicate assay balance and tightly wrapped in pure gold foil. This little packet was held in a charred splinter of wood and rapidly submerged in the molten gold. It was found better not to stir the gold, but to thoroughly mix the contents of the crucible by giving it a swinging motion".

The alloys were cast into lamp-black coated iron moulds to the form of bars $0.295 \times 0.250$ inches in cross-section and about $3 \frac{1}{2}$ inches long. The amounts of each impurity added was about 0.2 per cent.

Roberts-Austen would have liked to measure the effects of the 17 alloying additions on the melting point of gold but at the time, as he wrote, "slight variations in high melting points are very difficult to determine with any approach to accuracy". Within the next three years he was to develop methods of pyrometry which served to lay the foundations of the science of alloys but for the time being "it appeared to be better to ascertain the effect of metallic and other impurity on the cohesion of gold as indicated by the amount of force externally applied in an ordinary testing machine, and in that way to ascertain whether the effect of added metals is amenable to any known law". 
The tests were accordingly made on the small cast bars without further manipulation or machining, measurement being recorded of breaking strength and elongation on 3 inches. The results, plotted against calculated atomic volumes of the added impurity element, are shown in the graphs from the original paper.

It will be seen that silver and palladium have only a slight strengthening effect, while zinc, rhodium, manganese, indium, copper, lithium and aluminium produce progressively greater strengthening in that order. The weakening effect of the other elements is much more marked and more closely related to their increasing atomic volumes.

The paper attracted considerable attention, particularly among engineers who were becoming increasingly concerned about the lack of understanding of the properties of steels and other constructional materials. Thus it came about that Roberts-Austen was appointed by the Alloys Research Committee to determine whether the principle that "the larger the volume of the added atoms the greater the effect on the gold" would apply also to the properties of iron, copper and lead alloys.

The first report, published in October 1891, is remarkable for describing the first application of the Le Chatelier thermocouple (platinum with 10 per cent rhodium-platinum alloy) to metallurgical investigations and particularly for the development of an autographic recorder for observing the heating and cooling of alloy samples. Roberts-Austen immediately applied the recorder to determine the "atomic fall" in the melting point of alloys of gold with 1 atomic per cent of various additions. The values he found were as follows:

\begin{tabular}{lccc} 
& \multicolumn{3}{c}{ Atomic fall deg C } \\
Lead .. & $\ldots$ & $\ldots$ & 13 \\
Bismuth & $\ldots$ & $\ldots$ & 17 \\
Platinum & $\ldots$ & $\ldots$ & 17 \\
Silicon & $\ldots$ &. & 16
\end{tabular}

When he studied the effect of silver additions he found that it was necessary to add nearly 5 per cent before any lowering of the freezing point could be observed. At the time this caused him to wonder whether any silver at all "dissolved" in gold!

The first report was quite amazingly discursive but, as Dr Sidney W. Smith has noted, "the very discursiveness of the earlier reports provoked an amount of discussion from all sources which in no small measure contributed to make the work known and appreciated".

Among those metallurgists who were critical of the choice of gold alloys in this work was J. O. Arnold, a young man of 33 not long appointed Professor of Metallurgy at the University of Sheffield. $\mathrm{He}$ questioned whether the plan did not entail a great waste of precious labour. Such comparatively simple substances as gold and lead seemed to him not likely to furnish any useful analogies for investigating so marvellously complex a subject as steel. Steel research (which all this work was intended to preface) was in his opinion a field of too national an importance "to be used lightly as a cantering ground for the hobbies of periodicity and allotropy".

Roberts-Austen dealt kindly with him, wishing, he said, to say nothing that would discourage anyone at the outset of his career, and pointed out mildly that the terms of reference of the Committee were in fact to study the effects of certain elements on iron, copper and lead. In later years Arnold came to be among the greatest admirers of Roberts-Austen.

From these simple beginnings, the next four Reports published in 1893, 1895, 1897 and 1899 went on to range over almost the whole field of physical metallurgy. Questions of allotropy, oxidation, the effects of impurities in best select copper, the characteristics of some intermetallic compounds and the phenomenon of diffusion between solid metals were among those examined, and the use of freezing-point curves as a means of studying alloy systems was developed. The first copper-tin and copper-zinc curves were established and a start was made on the complex and important carbon-iron system.

Towards the end of his life Roberts-Austen adopted and made good use of the microscopical techniques devised by Sorby and, independently, by Martens. He applied them in the first instance to the study of steels for coinage dies and published his first photomicrographs in the annual report of the Royal Mint for 1896. In the same year, 1896, RobertsAusten summarised the advances made in understanding the structure of metals during the six years for which the Alloys Research Committee had been in existence in the course of a joint paper with $\mathrm{F}$. Osmond presented to the Royal Society; and here, in almost his final work, he illustrated their findings with photomicrographs of the identical series of gold alloys he had studied in 1888 with such notable results.

Roberts-Austen's work, so initiated, stands as an inspiration to all metallurgists. His biographer, Sidney W. Smith, wrote, "He clearly understood that useful results are only achieved by beginning somewhere and doing things. In this way he revealed to himself unsuspected aspects of the problems he was engaged upon. Much of Roberts-Austen's earlier work was in this sense a groping for principles which by their elusiveness led him to other and wider fields. He put in "section posts", leaving to others the work of surveying the land more completely".

And it all started with seventeen small gold alloy bars. 